\title{
Division of Clinical Sciences
}

National Cancer Institute

\section{Source}

National Cancer Institute. Division of Clinical Sciences. NCI Thesaurus. Code C18752.

Merged with the Division of Basic Sciences in January, 2001 to form the Center for Cancer Research 